\title{
Undiagnosed liver diseases
}

\author{
Emily Gao ${ }^{1 \#}$, Julian Hercun ${ }^{2 \#}$, Theo Heller ${ }^{2}$, Sílvia Vilarinho ${ }^{1,3}$
}

${ }^{1}$ Department of Internal Medicine, Section of Digestive Diseases, Yale School of Medicine, New Haven, CT, USA; ${ }^{2}$ Translational Hepatology Section, National Institute of Diabetes \& Digestive \& Kidney Diseases, National Institute of Health, Bethesda, MD, USA; ${ }^{3}$ Department of Pathology, Yale School of Medicine, New Haven, CT, USA

Contributions: (I) Conception and design: All authors; (II) Administrative support: None; (III) Provision of study materials or patients: None; (IV) Collection and assembly of data: None; (V) Data analysis and interpretation: None; (VI) Manuscript writing: All authors; (VII) Final approval of manuscript: All authors.

"These authors contributed equally to this work.

Correspondence to: Theo Heller, MD. Liver Diseases Branch, National Institute of Diabetes \& Digestive \& Kidney Diseases, National Institute of Health, Building 10, Room 10N248A 10 Center Drive, Bethesda, MD, 20814, USA. Email: theo.heller@nih.gov; Silvia Vilarinho, MD, PhD. Departments of Internal Medicine (Digestive Diseases) and of Pathology, Yale School of Medicine, 333 Cedar Street, LMP1080, New Haven, CT 06510, USA. Email: silvia.vilarinho@yale.edu.

\begin{abstract}
The landscape of chronic liver disease has drastically changed over the past 20 years, largely due to advances in antiviral therapy and the rise of metabolic syndrome and associated non-alcoholic fatty liver disease (NAFLD). Despite advances in the diagnosis and treatment of a variety of liver diseases, the burden of chronic liver disease is increasing worldwide. The first step to addressing any disease is accurate diagnosis. Here, we discuss liver diseases that remain undiagnosed, either because they are difficult to diagnose or due to hepatic manifestations of an unrecognized systemic disease. Additionally, their underlying etiology may remain unknown or they represent previously uncharacterized and therefore novel liver diseases. Our goal is to provide a framework for approaching undiagnosed liver diseases which elude standard hepatic diagnostic work-up and whose patterns of disease are often overlooked.
\end{abstract}

Keywords: Idiopathic liver disease; cryptogenic cirrhosis (CC); unmet medical need

Received: 10 December 2019; Accepted: 19 March 2020; Published: 05 April 2021.

doi: $10.21037 / \operatorname{tgh} .2020 .04 .04$

View this article at: http://dx.doi.org/10.21037/tgh.2020.04.04

\section{Introduction}

Chronic liver disease is the $11^{\text {th }}$ leading cause of death in the US and its incidence has been rising in the developing world with no signs of abatement (1-3). Many patients with abnormal liver function tests are asymptomatic, making the prevalence of liver dysfunction among the general population difficult to ascertain. In a retrospective study of 354 adult patients with abnormal liver function tests who underwent liver biopsy, $6 \%$ of patients had normal liver histology and $9 \%$ of patients had hepatitis of unknown etiology (4). Moreover, a prospective study of 272 adults with 6 months of persistently abnormal liver function tests identified no etiology for such abnormalities in nearly $20 \%$ of this group of patients (5). Altogether, these studies illustrate our incomplete understanding of the overall prevalence of liver dysfunction; and at this point in time, the spectrum of molecular pathogenesis and natural history of liver disease remain unexplained in a significant number of patients.

The absence of a definitive diagnosis indicates a lack of understanding as to why an individual develops liver disease, which severely limits appropriate treatment and prognostication for these patients. Firstly, when studying idiopathic liver disease, many studies tend to agglomerate all cases of idiopathic liver disease into a single group. Such studies compare the demographics, clinical features and outcomes of individuals with undiagnosed liver disease to individuals with a well-defined diagnosis of liver disease (6-9). Secondly, many of these comparative studies tend 
Table 1 A proposed approach to undiagnosed liver disease

\begin{tabular}{|c|c|}
\hline 1. A hidden liver disease & Is it an underdiagnosis or a misdiagnosis of a well-characterized hepatic disease? \\
\hline 2. A disguised liver disease & $\begin{array}{l}\text { Could it be a hepatic manifestation of a well-characterized disease better known for its } \\
\text { extra-hepatic presentation (i.e., cystic fibrosis)? }\end{array}$ \\
\hline 3. A known liver disease of unknown cause & Is it a well-characterized liver phenotype with unknown etiology (i.e., biliary atresia, PSC)? \\
\hline 4. An unknown liver disease & $\begin{array}{l}\text { Could it be a novel liver disease with uncharacterized clinical features, natural history and } \\
\text { unknown cause? }\end{array}$ \\
\hline
\end{tabular}

to be limited to small case series (7-9). It is important to recognize that this approach is deeply flawed since it merges data from several diseases with distinct pathogeneses, creating an "average" of "diseases" that do not exist and therefore inapplicable to patient care.

Cryptogenic cirrhosis (CC) defined as cirrhosis of unknown etiology after unraveling extensive testing (10) exemplifies this concept. The decreasing prevalence of CC over the past 40 years reflects the progress made in diagnosing idiopathic liver disease (11). Specifically, the discovery of hepatitis B virus (HBV) in 1965, hepatitis C virus (HCV) in 1989 and the concomitant development of serological testing, led to the recognition that viral hepatitis infections were responsible for a large number of cases of cirrhosis. Moreover, improvement in the definition and identification of autoimmune hepatitis in the last three decades and increasing recognition of non-alcoholic fatty liver disease (NAFLD) as a cause of hepatic dysfunction further explained additional cases of cirrhosis. Today, we all recognize that each etiology (viral, autoimmune, among others) has its own distinct treatment and overall prognosis $(6,12)$.

Here, we examine several broad categories of undiagnosed liver disease, which span from atypical presentations of known diseases to uncharacterized liver disorders. Specifically, we describe four broad categories of undiagnosed liver disease: (I) the hidden liver disease, which consists of underdiagnosis or misdiagnosis of wellcharacterized hepatic disease; (II) the disguised liver disease, which includes hepatic manifestations of broader well-characterized diseases better known for extrahepatic presentation (i.e., cystic fibrosis); (III) the known liver disease of unknown cause, such as well-characterized liver phenotypes with unknown etiology [i.e., biliary atresia (BA)], and (IV) the unknown liver diseases, encompassing novel liver diseases with uncharacterized phenotypic features, natural history and unknown cause (Table 1). Lastly, we will also discuss undiagnosed stage of liver disease and provide recommendations regarding how to approach liver diseases of unknown etiology.

\section{The hidden known liver diseases}

Liver disease can remain undiagnosed and undetected until clinical manifestations become apparent. Common liver diseases, such as viral hepatitis and NAFLD, can remain silent for decades. These known liver diseases often evade routine detection and their diagnosis depends on improved screening and awareness. Additionally, liver related mortality due to viral hepatitis might be underreported $(13,14)$. This well-recognized cause of liver disease can have severe consequences in at risk populations, including immunocompromised and chronic liver disease patients.

Viral hepatitis has the potential to remain silent due to underdiagnosis resulting from failure to screen in appropriate populations or from false-negative screening tests. Although major advances in the field of chronic $\mathrm{HCV}$ infection over the last three decades have led to safe curative therapies for millions of infected people (15) and increased public awareness, testing for $\mathrm{HCV}$ is undertaken far too infrequently. In the primary care setting, there is concerning data showing that only $16 \%$ of patients with consistently elevated transaminases underwent serological testing for $\mathrm{HCV}$ (16).

Hepatitis D virus (HDV) is also routinely undertested. In a cohort of $\mathrm{HBV}$ positive American veterans only 7.8\% were co-tested for HDV (17), while in a European cohort only $30 \%$ of HBV positive patients were co-tested (18), despite recommendations for more widespread HDV testing. Nonetheless, even if appropriate screening in at risk patients is performed, there is a possibility of false negatives. For example, occult HBV infection, defined as detectable HBV DNA in HBV surface antigen ( $\mathrm{HBsAg}$ ) negative individuals, remains a public health danger in $\mathrm{HBV}$ endemic 
regions such as Africa and Asia, where recent population studies suggest a prevalence of 8-26\% (19-23).

Failure to screen for or to detect viral hepatitis occurs not only in asymptomatic patients but can lead to missed diagnoses or misdiagnosis in patients with clinically active liver disease, as exemplified by hepatitis E virus (HEV) infection. Although HEV testing is often not routinely performed in the US due to low suspected prevalence of the disease, viral testing and causality assessment among a cohort of 318 patients suspected to have drug-induced liver injury (DILI) found that $3 \%$ of acute liver injuries misdiagnosed as DILI were more likely HEV infections (24). A smaller cohort in the UK found that $12 \%$ of patients suspected of DILI were likely acutely infected with HEV (25). In another cohort of patients with suspected viral hepatitis of unknown etiology, 30\% had HEV viremia (26). Underscreening for $\mathrm{HEV}$ is likely due to previous assumptions that $\mathrm{HEV}$ infection is restricted to endemic regions and exclusively a cause of acute hepatitis, though today we know that both these assumptions are untrue (27). HEV genotype 3, an endemic zoonosis in developed countries, has received increased attention since its first description in transplant patients in 2008 (28). In such patients, HEV infection has been implicated in causing chronic hepatitis with persistence of detectable viral load for more than 6 months. Solid organ transplant recipients (including liver transplant patients) are especially at risk for chronic $\mathrm{HEV}$ infection as $46 \%$ to $80 \%$ of them are unable to clear the virus spontaneously $(29,30)$. Furthermore, fibrosis can occur due to ongoing liver injury in those patients (30). A high prevalence of $\mathrm{HEV}$ (32\%) was noted in patients developing cirrhosis of the graft (31). An additional challenge in this population on chronic immunosuppression is the reliability of antibody titers; therefore reliance on RNA detection is recommended (29,32). Patients with chronic liver disease comprise another at risk group who are at increased likelihood of acute on chronic liver failure if infected with HEV (33-36). Given the fact that HEV infection may be more severe than previously thought in these select patient populations, transfusion transmitted $\mathrm{HEV}$ is an emerging concern. In the US, as well as in the majority of European Union countries, donated blood is not routinely screened for $\operatorname{HEV}(37,38)$. However, several studies have shown instances of transfusion-transmitted HEV infection $(39,40)$. Among a French cohort of 23 patients with symptomatic transfusion-transmitted $\mathrm{HEV}$ infection, $61 \%$, all on chronic immunosuppression, developed chronic infection (40). With asymptomatic cases potentially underrepresented and improving HEV RNA screening tools, many have advocated for a more widespread testing of blood products, as already established in several European countries including Ireland, France, Germany, the UK and the Netherlands $(37,38,41)$. Altogether, HEV infection should also be considered in individuals where other causes of liver disease have been ruled out.

\section{The disguised known liver diseases}

Liver involvement is increasingly being recognized as a major aspect of multisystemic diseases and its severity as an important prognostic marker. Previously unrecognized due to other predominant clinical features or due to limited life expectancy, liver involvement is uncovered in patients with diverse diseases when careful investigations are performed. Whereas systemic diseases may have subtle liver dysfunction overshadowed by extrahepatic features, systemic diseases with heterogeneous presentation may also present non-classically with liver involvement as a first and/or predominant clinical presentation. Being aware of hepatic phenotypes in systemic disease thus becomes relevant to clinicians, not only to diagnose systemic illness in patients with liver disease, but more importantly to understand the pathophysiology of liver dysfunction in such patients, which directly impact their management. This section will illustrate various presentations of disguised liver involvement in systemic diseases.

In some systemic illnesses, the site of the predominant clinical manifestation may disguise significant hepatic involvement. In autosomal recessive polycystic kidney disease (ARPKD), hepatobiliary involvement is often overshadowed by the severity of renal disease. However, the liver is invariably involved due to the presence of congenital hepatic fibrosis $(\mathrm{CHF})$, which can be overlooked since it may remain silent with preserved hepatic function. Nonetheless, the initial clinical presentation in up to one quarter of patients can be due to liver-related manifestations (42). In these cases, liver involvement is more apparent through manifestations of portal hypertension (splenomegaly, thrombocytopenia or esophageal varices) or cholangitis due to bile duct involvement $(42,43)$. For instance, in one cohort of 73 patients with ARPKD, $6 \%$ had cholangitis, and over $71 \%$ had signs of portal hypertension (42). Clinicians must be aware of the diverse manifestations of liver involvement in ARPKD as it is not associated with a specific mutation of PKHD1 (the causative gene for ARPKD) and is independent of the severity of 


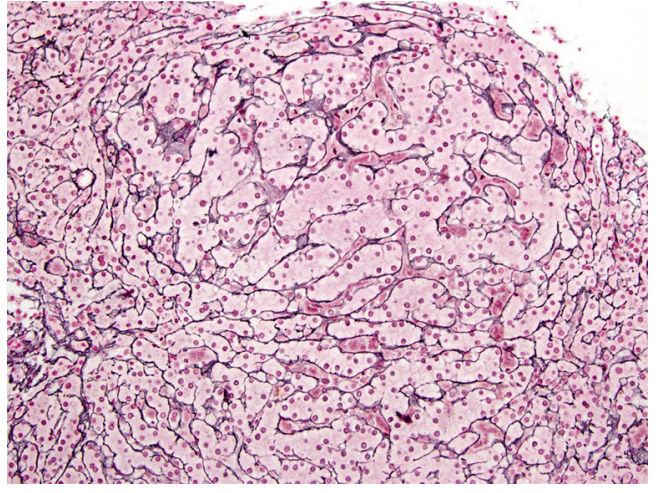

Figure 1 Reticulin stain of a liver biopsy from a patient with sickle cell disease demonstrating nodular regenerative hyperplasia (NRH). In the center of the slide, hyperplastic hepatocytes can be identified creating a nodule without any identifiable fibrosis. (Slide courtesy of Dr. David Kleiner). 200× magnification.

kidney disease (42).

Hepatic involvement can also be uncovered in multisystemic conditions due to increased life expectancy. For instance, our knowledge of cystic fibrosis related liver disease (CFLD) and its natural history has grown substantially over time due to the development of effective treatments that significantly increased the survival of patients. Initially viewed as a disease of childhood, CFLD is being increasingly recognized in adults with cystic fibrosis, with a subset of patients diagnosed with liver disease during adulthood. In a large French cohort, a yearly $1-2 \%$ increase in incidence in CFLD continued into early adulthood (44). CFLD presents with a wide range of clinical manifestations, including elevated liver enzymes and anomalies on imaging, which are taken into account in the consensus diagnostic criteria (45). However, a high index of suspicion is required as some cases are only diagnosed in the presence of complications due to advanced liver disease and portal hypertension. As in other conditions with a recent improvement in survival, cases of non-cirrhotic portal hypertension $(\mathrm{NCPH})$ have been described, challenging the widely accepted endpoint of biliary cirrhosis in CFLD $(46,47)$. The presence of liver involvement is an important prognostic marker in adult cystic fibrosis patients as it is independently associated with lung transplantation and death (48).

Another condition where improved management and greater life expectancy have led to increased recognition of liver involvement is sickle cell disease (SCD). Prior to the establishment of adequate screening of blood products, the requirement for multiple transfusions in this population increased the risk of viral hepatitis infections. However, other risk factors for liver disease and fibrosis remain, including transfusion-induced iron overload and chronic vaso-occlusive lesions (49). In adult patients, abnormal liver parameters (alkaline phosphatase, direct bilirubin and albumin) are associated with mortality (50). Initially described in case reports in the SCD population, auto-immune liver disease has now been reported in up to $17 \%$ of patients with liver disease in larger cohorts (49). Besides the previously mentioned risk factors for liver injury and fibrosis, liver involvement can also manifest itself through nodular regenerative hyperplasia (NRH) (Figure 1), described in $36 \%$ of SCD patients undergoing liver biopsy in one cohort (50). Cirrhosis has been found at autopsy in 11-14\% of patients (50).

In other conditions, such as primary immunodeficiencies, while liver involvement is a well recognized manifestation its clinical presentation and etiology remain broad and elusive. The reported prevalence of liver involvement in common variable immune deficiency (CVID) ranges from $9 \%$ to $79 \%(51,52)$. Like in other disorders discussed above, the presence of liver disease is an important prognostic marker $(52,53)$. Although historically the predominant cause of liver disease in CVID was transfusion-transmitted viral hepatitis (53), in light of improved viral screening of the blood supply new patterns of liver involvement in this population have been recognized. NRH and granulomatous liver disease are two common phenotypes of liver involvement in CVID patients. More specifically, the prevalence of NRH has been reported at $5 \%$ overall in CVID cohorts (54), but can be seen in up to $87 \%$ of patients with available liver histology (55). Portal hypertension is also reported as a predominant clinical aspect in NRH (55). Recurrence of NRH in patients with CVID post liver transplantation has been described, which appears to be more likely due to recurrence of disease rather than secondary to immunosuppression or vascular causes (56). Although the severity of liver disease in CVID is increasingly recognized and better defined, our understanding of the underlying systemic condition is still evolving. For instance, activated phosphoinositide 3-kinase $\delta$ syndrome, which was once considered part of CVID, has recently been uncovered due to whole-exome sequencing (WES). Liver involvement was described in 30\% of these patients, with NRH as its predominant manifestation (57).

While remaining a manifestation of liver disease on 
its own, autoimmune hepatitis has also been described in the context of primary immune deficiencies, especially if predominantly $\mathrm{T}$ cell abnormalities are present, including in cases of autoimmune regulator (AIRE) deficiency, FOXP3 deficiency causing X-linked (IPEX) syndrome and STAT1 mutations. However, while auto-antibodies are present in those conditions, their clinical and prognostic significance remain unknown (58). Interestingly, the presence of one liver disease does not exclude the possibility of another as demonstrated in a subset of patients with CVID presenting with evidence of autoimmune like hepatitis superimposed on findings consistent with NRH (54).

As previously alluded to, distinguishing between different types of hepatic involvement in systemic disease can have an impact on management and prognosis. Although liver abscess is the most common manifestation of liver involvement in chronic granulomatous disease (CGD), a wide spectrum of liver abnormalities have also been described, ranging from non-specific elevation in liver enzymes to cirrhosis with portal hypertension. In cases where liver histological analysis is available, virtually all patients (95\%) present portal and/or lobular chronic hepatitis (59). Vascular lesions are prevalent, including venopathy of the portal and centrilobular veins. Furthermore, NRH is once again a common finding and described in a quarter of biopsies and in half of autopsy cases (59). The development of portal hypertension in patients with CGD is associated with an increase in mortality. In one cohort, although death was due to infectious causes in all cases, a progressive decrease in platelet count was an independent predictor of mortality. Additionally, an increase in portal pressure potentially affected the propensity of patients with CGD to develop infections (60). Thus, adequate characterization of liver involvement is an important prognostic marker.

Unexplained liver disease can be a component of a new clinical syndrome but can also be uncovered as a result of a thorough investigation of previously described diseases. Deficiency of adenosine deaminase 2 (DADA2) was first described in patients with multisystemic features including early-onset lacunar strokes, polyarteritis nodosa, livedoid rash and intermittent fevers (61). While hepatosplenomegaly was observed in 6 out of 7 patients from the original cohort, identifying additional patients has led to better characterization of liver involvement and the description of portal hypertension in $16 \%$ of patients in a subsequent cohort (62). Similarly, while telomere diseases are best known for pulmonary fibrosis, various phenotypes of liver involvement have been found in $10-40 \%$ of patients $(63,64)$. In a cohort of patients with liver involvement, morbidity associated with lung disease and significant mortality on follow-up were noted. NRH has also been described in patients with telomere disease, with a variable prevalence in different studies (63).

\section{The known liver diseases of unknown cause}

There are a variety of broad liver disease entities classified according to conventional clinical, laboratory and/or pathological features, routinely used by hepatologists, that may have distinct underlying molecular pathogeneses. These include cases of cirrhosis, NCPH, NAFLD, BA, primary sclerosing cholangitis (PSC) among others.

As reviewed above, one of the broadest and oldest categories of idiopathic liver disease is CC. Interestingly, in a study published in 2011, where a cohort of patients initially diagnosed with CC between 1990-2009 was reanalyzed, a definitive diagnosis was attained in nearly $30 \%$ of these patients (65). A review of 135,000 liver transplants performed between 1988 and 2015 revealed that $7 \%$ of patients were labeled as "cryptogenic" (10). More recently, per the UNOS database, the prevalence of CC among patients listed for liver transplant between 2002-2016 was $\sim 4 \%$ (6). CC represents the end-stage of the natural history of a variety and heterogeneous etiologies that lead to liver inflammation and collagen deposition. Hence, patients currently diagnosed with CC may represent undiscovered etiologies of liver injury. However, a subset of cases may be incorrectly attributed to alcoholic or NAFLD when the clinical picture may be ambivalent and the underlying molecular pathogenesis is not fully investigated or unrevealing.

$\mathrm{NCPH}$ consists of portal hypertension in the absence of advanced fibrosis/cirrhosis, steatohepatitis and extrahepatic thrombosis of the portal or hepatic veins (66-68). Liver biopsies of these patients may show thickening and dilation of the main intrahepatic portal vein and obliterative venopathy of the peripheral portal vein branches in the absence of cirrhosis $(69,70)$. Other common findings on biopsy of NCPH are NRH, phlebosclerosis, periportal shunting, sinusoidal dilatation, and rudimentary portal tract $(71,72)$. NCPH has been associated with a broad range of infectious, immunological, toxic, pro-thrombotic, genetic diseases discussed above $(67,73)$, as well as exposure to certain medications, such as didanosine and azathioprine $(74,75)$. Interestingly, in rare cases, loss-of-function 
mutations in DGUOK (76) and $K C N N 3$ (77), in a recessive and dominant inheritance pattern, respectively, have been shown to cause NCPH, providing new mechanistic insights into this poorly understood liver disease.

NAFLD is classically associated with obesity, central adiposity and the metabolic syndrome $(78,79)$. However, triglyceride accumulation in the cytoplasm of hepatocytes can result from a variety of other etiologies, such as mitochondrial disorders, lipid and lipoprotein metabolism diseases, among others $(80,81)$. An obvious area of study to parse out the multiple contributors of NAFLD/NASH pathogenesis are the individuals who present with hepatic steatosis (>5\%) and are lean, generally defined as BMI $<25 \mathrm{~kg} / \mathrm{m}^{2}$ (82). Of note, the elevated rate of hepatic steatosis at lower BMI in Asian individuals have led some to propose an ethnic-specific lean NASH criteria of BMI $<23 \mathrm{~kg} / \mathrm{m}^{2}$ in Asian individuals (83-85), which may still be a "moving target" as we advance our understanding about the natural history of NAFLD in the context of obesity and metabolic syndrome. Whereas the prevalence of lean NAFLD in the US is estimated to be approximately $7 \%$ and lean NASH is estimated to be $1.4 \%$ (86), its prevalence appears to be higher in Asian populations. Studies in India, Japan, China and Korea showed a lean NAFLD and NASH prevalence of $8-27 \%(87-89)$ and $2 \%$ respectively (90-93). It remains poorly understood why only $5-10 \%$ of patients with NAFLD develop sterile inflammation: NASH $(81,94)$, and who are the $10-40 \%$ of patients with $\mathrm{NASH}$ that will progress to advanced fibrosis, cirrhosis and hepatocellular carcinoma $(95,96)$. Moreover, whether or not NAFLD and NASH in lean individuals has worse, similar or better clinical outcomes compared to obese patients with NAFLD and NASH remains controversial (97-100). A likely explanation for the current conflicting data on this topic is that this group includes a diverse molecular etiology of NAFLD/NASH $(80,101)$ with a distinct natural history. For instance, it will be informative to follow longitudinally patients with heterozygous familial hypobetalipoproteinemia due to loss-of-function mutations in $A P O B(101,102)$, a genetic disorder which manifests with NAFLD in the context of hypolipidemia.

A classic example of a well-characterized disease of unknown etiology is PSC. PSC results from progressive inflammation and fibrosis of bile ducts, which leads to intrahepatic and extrahepatic biliary strictures, impaired bile formation and drainage. PSC patients are at increased risk for end-stage liver disease and hepatobiliary cancers and represent an underserved population with unmet medical needs. In fact, therapeutic options are limited and at least one fourth of PSC patients requiring liver transplantation develop disease recurrence in the graft. Though the cause of PSC is unknown, contributing factors are theorized to include genetic predisposition, environmental factors and microbiota triggers, with gut-liver dysbiosis resulting in autoimmune disease. However, the autoimmune theory of PSC pathogenesis is belied by the lack of efficacy in immunosuppressive agents in controlling the disease, reinforcing that our understanding of pathogenesis is incomplete (103-105).

Another classic cause of liver disease of unknown etiology is BA. BA is a congenital obstructive cholangiopathy and if untreated will result in death from liver failure within the first 2 years of life (106). BA is the leading cause of neonatal cholestasis, accounting for over $40 \%$ of pediatric liver transplants worldwide (107). Despite extensive research efforts over the last decades, its pathogenesis remains unknown, which hinders the development of non-invasive diagnostic and therapeutic tools. Like other liver diseases with unknown etiology, such as PSC, it is possible that BA represents a clinical and histopathologic pattern of distinct molecular liver diseases.

\section{The unknown liver diseases}

Epidemiological patterns and associations give us clues to the currently undiscovered causes of liver disease. Historically, viral infection has been a major source of chronic liver disease previously labeled as idiopathic. In the last decade, due to major advances in human genomics through next generation sequencing technologies, unrecognized genetic liver diseases present another etiology of idiopathic liver disease ripe for further exploration. In addition to discovering novel genetic causes of primary liver disease, these sequencing technologies also have a broader role in uncovering additional infectious agents underlying liver diseases. For instance, WES of 193 hepatocellular carcinoma tissue specimens showed that $5.7 \%$ had clonal insertions in cancer drivers by adeno-associated virus 2 (AAV2), a virus considered to be nonpathogenic up to this study (108).

Over the last four decades, genetic discoveries in liver disease have ranged from identification of rare genetic variants causal of monogenic liver diseases to the recognition of common single nucleotide polymorphisms associated with increased or protective risk for liver disease. Based on the Online Mendelian Inheritance in Man (OMIM) 


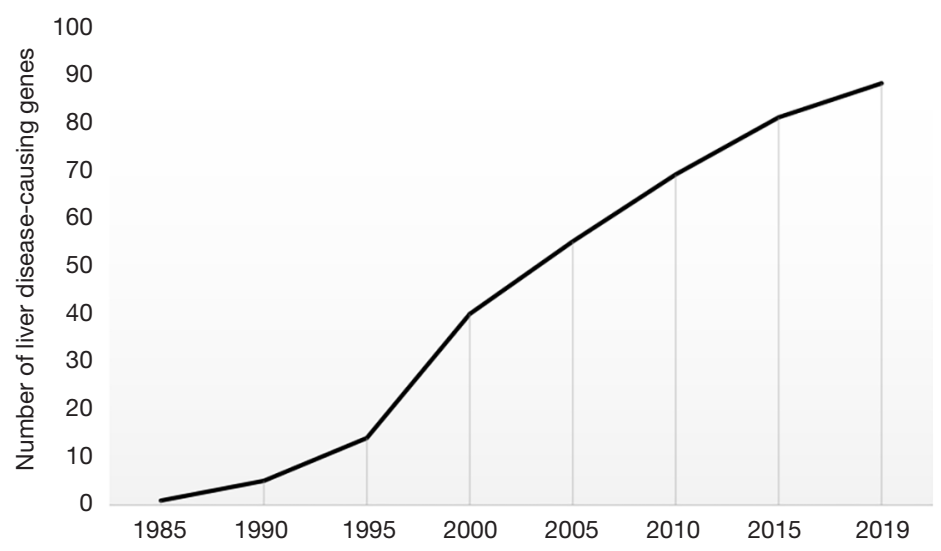

Figure 2 Representation of the rising number of liver disease-causing genes discovered since 1985 based on OMIM (Online Mendelian Inheritance in Man) data (last accessed in August 31, 2019). Specifically, genes and phenotypes associated with "cirrhosis" were searched in the OMIM database. These genes associated with liver disease were further investigated in pubmed to find the original discovery date. Somatic mutations, gene associations, and cases in which there was limited evidence of liver disease-gene causality were removed.

database, there are at least 88 monogenic diseases that have significant liver involvement as defined by cirrhosis (last accessed on August 31, 2019). Over 50\% of these genetic diseases were discovered within the past 20 years, and nearly $25 \%$ of them were discovered within the past 10 years (Figure 2). In fact, it has only been a decade since WES was first demonstrated to be a very effective tool for clinical diagnosis and human disease gene discovery. On the other hand, genome wide association studies (GWAS) have shown an association between PNPLA3 variant (p.I148M) and alcoholic and NAFLD (109,110). More importantly, individual carriers of this risk allele are at a significant higher risk of developing advanced liver fibrosis as their $\mathrm{BMI}$ and hepatic triglyceride content increase, but their risk for NAFLD is not much different from non-PNPLA3 variant carriers if their BMI is within normal range (111). More recently, a very large study revealed the first association between a truncating mutation in HSD17B13 and protection against chronic liver disease (112), including in individuals who are carriers of the PNPLA3 risk allele.

Collectively, advances in genetics and genomics of liver disease provide new insights into the underlying molecular pathogenesis, building on clinical and histological phenotypes and redefining the current taxonomy of liver disease (113). Historically, HBV and HCV infections illustrate how focusing on disease pathogenesis, rather than exclusively on clinical presentation and histological findings, can lead to novel therapies with enormous benefits to the patient. It is important to recognize the limitations of phenotypic categorization alone as liver biopsies in patients with idiopathic liver disease can have non-specific findings showing downstream sequelae of chronic liver inflammation, cirrhosis or NRH rather than revealing the initial trigger (etiology) of disease $(67,114)$. Moreover, patients with similar genetic liver disease may have heterogeneous clinical presentations.

\section{Undiagnosed stage of liver disease}

Assessing the degree of liver involvement is crucial in determining the prognosis of liver disease. In community and hospital based studies, $16.5 \%$ of patients with chronic $\mathrm{HCV}$ managed in primary care have advanced fibrosis; an underestimation of the proportion of patients with advanced fibrosis in the community setting can have a significant impact on liver related outcomes (115). As this discrepancy can occur in situations where surrogate markers are available and well validated, staging the severity of liver involvement and of portal hypertension with non-invasive measures is an even greater challenge in rare and unknown diseases. Not only are non-invasive markers not validated, but also some of the commonly used clinical surrogates are potentially inadequate. For instance, splenomegaly is part of the usual clinical manifestations in telomere disease (63) and in CVID (where its prevalence reaches 40\%) (116), therefore its presence cannot be solely attributed to portal hypertension. On the other hand, autosplenectomy in sickle 
cell disease renders platelet counts less reliable (50).

Encouragingly, in some conditions such as CFLD, the use of non-invasive testing has become more frequent and can be integrated in diagnostic algorithms and used to determine the stage of the disease (46). Readily accessible serological markers can also be of use in other conditions. Progressive decrease in platelet count correlates with mortality in adult patients with CFLD (46) and in CGD (60). In SCD, elevation in bilirubin is common and often thought to be related to hemolysis. However, direct bilirubin levels correlate with primary bile acid levels, confirming it can be used as a surrogate marker for liver function in this population (50).

Diverse conditions in which NRH is a histologic manifestation of liver involvement are often studied together when portal hypertension and prognosis are evaluated. In NRH, usual non-invasive markers of fibrosis, which use have been validated in cirrhosis for staging of liver disease and predicting complications, can be abnormal but do not have a good correlation with the severity of portal hypertension $(117,118)$. For many years, NRH was thought to be associated with a benign clinical course, but in the subset of patients with non-cirrhotic portal hypertension, a potentially more rapid progression to hemorrhagic portal hypertensive complications than in patients with cirrhosis has been described (119). The presinusoidal pattern of portal hypertension in NRH renders hepatic venous pressure gradient (HVPG) measurements unreliable in determining the risk of complications related to portal hypertension $(120,121)$. While portal vein pressure is considered a more reliable indicator, it is seldom obtained for diagnostic purposes. In multiple studies of patients with NRH and clinically significant portal hypertension (including variceal hemorrhage), the average HVPG $(7.0-7.6 \mathrm{mmHg})(118,121,122)$ was below the thresholds established for clinically significant portal hypertension associated with cirrhosis (HVPG >10 mmHg) (121-124). Of note, some patients present with an HVPG $>10 \mathrm{mmHg}$ (up to $25 \%$ in one cohort) (123) and when HVPG is increased a correlation has been found with the spleen and liver volumes (125). These findings highlight the need for surrogate markers of portal hypertension in NRH and a high degree of clinical suspicion for portal hypertension when evaluating these patients.

\section{Proposed approach to liver disease of unknown etiology}

Liver disease of unknown etiology remains a major unmet medical need both in adult and pediatric hepatology practices. In addition to improving screening and detection of known liver diseases, it is important to continue investigating the molecular causes of unexplained liver dysfunction. The study of unexplained illness and atypical clinical presentations of a broad array of human diseases phenotypes underlies several major advances in basic science, disease pathobiology and therapeutics (126). Hence, recognizing and investigating molecular subtypes of liver diseases will lead not only to a better understanding of why patients develop liver disease but also will advance our current knowledge of liver function in health and disease. These discoveries have the potential to uncover novel pathways for medical intervention and therapeutic design for both rare and common liver diseases.

Collectively, this review explores how the same clinicpathological diagnoses (e.g., NAFLD, NASH, NCPH...) can have distinct molecular pathogenesis and therefore represent different diseases. Since WES is now available in the clinical setting, its incorporation in the algorithm of evaluation of children and adults with liver disease of unknown etiology should be considered $(80,127,128)$. A future where nearly all individuals seeking medical care would have his/her genome sequenced would provide broad information regarding which diseases individuals are at risk for, why they became sick and how to best treat them. Multidisciplinary Genome Rounds to discuss and integrate each patient's genotype and liver phenotype information have been proposed $(80,113)$. Furthermore, given the pace of genetic discovery, review of the genetic results of a patient who remains undiagnosed should be iterative with annual re-analyses to account for new discoveries (129). Several studies have shown that $7-10 \%$ of patients who remained undiagnosed after exome sequencing received a diagnosis on re-analysis of genetic data at a later date $(127,129)$, largely attributed to case reports discovered after the patient's initial evaluation. The study and investigation of idiopathic liver diseases are important, not solely for the underserved patients who suffer from these poorly understood diseases, but also for furthering our understanding of normal liver function. Ultimately, this approach could stimulate the development of new effective non-invasive diagnostic and therapeutic tools applicable to more common liver diseases.

The description of new clinical entities, better awareness of known liver disease, improved recognition of atypical or subtle hepatic involvement in systemic diseases will advance knowledge and improve patient care. Manifestations of liver disease deemed idiopathic decades ago are well- 
defined clinical syndromes today. Thus, by integrating and expanding the knowledge outlined above, the liver phenotypes that remain unexplained today will be easily diagnosed and hopefully treated in years to come.

\section{Acknowledgments}

Funding: SV is supported by the National Institute of Diabetes and Digestive and Kidney Diseases of the National Institutes of Health under Award Number K08 DK113109. The content is solely the responsibility of the authors and does not necessarily represent the official views of the National Institutes of Health.

\section{Footnote}

Provenance and Peer Review: This article was commissioned by the Guest Editors (Luca Fabris and Mario Strazzabosco) for the series "Recent Advances in Rare Liver Diseases" published in Translational Gastroenterology and Hepatology. The article was sent for external peer review organized by the Guest Editors and the editorial office.

Conflicts of Interest: All authors have completed the ICMJE uniform disclosure form (available at http://dx.doi. org/10.21037/tgh.2020.04.04). The series "Recent Advances in Rare Liver Diseases" was commissioned by the editorial office without any funding or sponsorship. The authors have no other conflicts of interest to declare.

Ethical Statement: The authors are accountable for all aspects of the work in ensuring that questions related to the accuracy or integrity of any part of the work are appropriately investigated and resolved.

Open Access Statement: This is an Open Access article distributed in accordance with the Creative Commons Attribution-NonCommercial-NoDerivs 4.0 International License (CC BY-NC-ND 4.0), which permits the noncommercial replication and distribution of the article with the strict proviso that no changes or edits are made and the original work is properly cited (including links to both the formal publication through the relevant DOI and the license). See: https://creativecommons.org/licenses/by-nc-nd/4.0/.

\section{References}

1. Heron MP. Deaths: leading causes for 2016. 2018.
2. Health, Unnited States 2015 - Individual Charts and Tables: Spreadsheet, PDF, and Powerpoint files. In: National Center of Health Statistics. Centers for Disease Control and Prevention. Available online: http://www.cdc. gov/nchs/hus/contents2015.htm\#017. Accessed September 5, 20192019.

3. Heron MP. Deaths: leading causes for 2017. 2019.

4. Skelly MM, James PD, Ryder SD. Findings on liver biopsy to investigate abnormal liver function tests in the absence of diagnostic serology. J Hepatol 2001;35:195-9.

5. de Lédinghen V, Ratziu V, Causse X, et al. Diagnostic and predictive factors of significant liver fibrosis and minimal lesions in patients with persistent unexplained elevated transaminases. A prospective multicenter study. J Hepatol 2006;45:592-9.

6. Thuluvath PJ, Kantsevoy S, Thuluvath AJ, et al. Is cryptogenic cirrhosis different from NASH cirrhosis? J Hepatol 2018;68:519-25.

7. Ayata G, Gordon FD, Lewis WD, et al. Cryptogenic cirrhosis: Clinicopathologic findings at and after liver transplantation. Human Pathology 2002;33:1098-104.

8. Tardu A, Karagul S, Yagci MA, et al. Histopathological Examination of Explanted Liver After Transplantation in Patients With Cryptogenic Cirrhosis. Transplant Proc 2015;47:1450-2.

9. Yalamanchili K, Saadeh S, Klintmalm GB, et al. Nonalcoholic fatty liver disease after liver transplantation for cryptogenic cirrhosis or nonalcoholic fatty liver disease. Liver Transpl 2010;16:431-9.

10. Mercado-Irizarry A, Torres EA. Cryptogenic cirrhosis: Current knowledge and future directions. Clin Liver Dis (Hoboken) 2016;7:69-72.

11. Czaja AJ. Cryptogenic Chronic Hepatitis and Its Changing Guise in Adults. Dig Dis Sci 2011;56:3421-38.

12. Ludwig J, Viggiano TR, McGill DB, et al. Nonalcoholic steatohepatitis: Mayo Clinic experiences with a hitherto unnamed disease. Mayo Clin Proc 1980;55:434-8.

13. Vong S, Bell BP. Chronic liver disease mortality in the United States, 1990-1998. Hepatology 2004;39:476-83.

14. Thomas AR, Zaman A, Bell BP. Deaths from chronic liver disease and viral hepatitis, Multnomah County, Oregon, 2000. J Clin Gastroenterol 2007;41:859-62.

15. Vilarinho S, Lifton RP. Pioneering a Global Cure for Chronic Hepatitis C Virus Infection. Cell 2016;167:12-5.

16. Schreiner AD, Bian J, Zhang J, et al. The Association of Abnormal Liver Tests with Hepatitis C Testing in Primary Care. Am J Med 2020;133:214-21.e1.

17. Kushner T, Serper M, Kaplan DE. Delta hepatitis 
within the Veterans Affairs medical system in the United States: Prevalence, risk factors, and outcomes. J Hepatol 2015;63:586-92.

18. Manesis EK, Vourli G, Dalekos G, et al. Prevalence and clinical course of hepatitis delta infection in Greece: a 13year prospective study. J Hepatol 2013;59:949-56.

19. Akintule OA, Olusola BA, Odaibo GN, et al. Occult HBV Infection in Nigeria. Arch Basic Appl Med 2018;6:87-93.

20. Minuk GY, Sun DF, Uhanova J, et al. Occult hepatitis B virus infection in a North American community-based population. J Hepatol 2005;42:480-5.

21. Bhattacharya H, Bhattacharya DD, Roy S, et al. Occult hepatitis $\mathrm{B}$ infection among individuals belonging to the aboriginal Nicobarese tribe of India. J Infect Dev Ctries 2014;8:1630-5.

22. Dickson RC, Everhart JE, Lake JR, et al. Transmission of hepatitis B by transplantation of livers from donors positive for antibody to hepatitis B core antigen. The National Institute of Diabetes and Digestive and Kidney Diseases Liver Transplantation Database. Gastroenterology 1997;113:1668-74.

23. Seo DH, Whang DH, Song EY, et al. Occult hepatitis B virus infection and blood transfusion. World J Hepatol 2015;7:600-6.

24. Davern TJ, Chalasani N, Fontana RJ, et al. Acute hepatitis E infection accounts for some cases of suspected drug-induced liver injury. Gastroenterology 2011;141:1665-72.e1-9.

25. Dalton HR, Fellows HJ, Stableforth $W$, et al. The role of hepatitis E virus testing in drug-induced liver injury. Aliment Pharmacol Ther 2007;26:1429-35.

26. Viera-Segura O, Realpe-Quintero M, Panduro A, et al. First detection of hepatitis $\mathrm{E}$ virus genotype 3 as a common infectious agent in patients with chronic liver damage in Mexico. Ann Hepatol 2019;18:571-7.

27. Webb GW, Dalton HR. Hepatitis E: an underestimated emerging threat. Ther Adv Infect Dis 2019;6:2049936119837162.

28. Kamar N, Selves J, Mansuy JM, et al. Hepatitis E virus and chronic hepatitis in organ-transplant recipients. N Engl J Med 2008;358:811-7.

29. Kamar N, Garrouste C, Haagsma EB, et al. Factors associated with chronic hepatitis in patients with hepatitis $\mathrm{E}$ virus infection who have received solid organ transplants. Gastroenterology 2011;140:1481-9.

30. Chauhan A, Webb G, Ferguson J. Clinical presentations of Hepatitis E: A clinical review with representative case histories. Clin Res Hepatol Gastroenterol 2019;43:649-57.

31. Riveiro-Barciela M, Buti M, Homs M, et al. Cirrhosis, liver transplantation and $\mathrm{HIV}$ infection are risk factors associated with hepatitis E virus infection. PLoS One 2014;9:e103028.

32. Owada Y, Oshiro Y, Inagaki Y, et al. A Nationwide Survey of Hepatitis E Virus Infection and Chronic Hepatitis in Heart and Kidney Transplant Recipients in Japan. Transplantation 2020;104:437-44.

33. Borkakoti J, Hazam RK, Kar P. Hepatitis E Virus: A Hidden Danger in Acute on Chronic Liver Failure. J Hepatol 2013;58:S499-S500.

34. Albano L, Vasco M, Benincasa G, et al. Pathogenic mechanisms and current epidemiological status of $\mathrm{HEV}$ infection in asymptomatic blood donors and patients with chronic diseases. Eur J Clin Microbiol Infect Dis 2019;38:1203-9.

35. Hamid SS, Atiq M, Shehzad F, et al. Hepatitis E virus superinfection in patients with chronic liver disease. Hepatology 2002;36:474-8.

36. Kumar Acharya S, Kumar Sharma P, Singh R, et al. Hepatitis $\mathrm{E}$ virus (HEV) infection in patients with cirrhosis is associated with rapid decompensation and death. $\mathrm{J}$ Hepatol 2007;46:387-94.

37. Domanović D, Tedder R, Blümel J, et al. Hepatitis E and blood donation safety in selected European countries: a shift to screening? Euro Surveill 2017;22:30514.

38. Boland F, Martinez A, Pomeroy L, et al. Blood Donor Screening for Hepatitis E Virus in the European Union. Transfus Med Hemother 2019;46:95-103.

39. Huzly D, Umhau M, Bettinger D, et al. Transfusiontransmitted hepatitis E in Germany, 2013. Euro Surveill 2014;19:20812.

40. Gallian P, Pouchol E, Djoudi R, et al. TransfusionTransmitted Hepatitis E Virus Infection in France. Transfus Med Rev 2019;33:146-53.

41. Denner J, Pischke S, Steinmann E, et al. Why all blood donations should be tested for hepatitis $\mathrm{E}$ virus (HEV). BMC Infect Dis 2019;19:541.

42. Gunay-Aygun M, Font-Montgomery E, Lukose L, et al. Characteristics of congenital hepatic fibrosis in a large cohort of patients with autosomal recessive polycystic kidney disease. Gastroenterology 2013;144:112-21.e2.

43. Burgmaier K, Kilian S, Bammens B, et al. Clinical courses and complications of young adults with Autosomal Recessive Polycystic Kidney Disease (ARPKD). Sci Rep 2019;9:7919.

44. Boëlle PY, Debray D, Guillot L, et al. Cystic Fibrosis Liver Disease: Outcomes and Risk Factors in a Large Cohort of French Patients. Hepatology 2019;69:1648-56. 
45. Debray D, Kelly D, Houwen R, et al. Best practice guidance for the diagnosis and management of cystic fibrosis-associated liver disease. J Cyst Fibros 2011;10 Suppl 2:S29-36.

46. Koh C, Sakiani S, Surana P, et al. Adult-onset cystic fibrosis liver disease: Diagnosis and characterization of an underappreciated entity. Hepatology 2017;66:591-601.

47. Witters P, Libbrecht L, Roskams T, et al. Liver disease in cystic fibrosis presents as non-cirrhotic portal hypertension. J Cyst Fibros 2017;16:e11-3.

48. Chryssostalis A, Hubert D, Coste J, et al. Liver disease in adult patients with cystic fibrosis: a frequent and independent prognostic factor associated with death or lung transplantation. J Hepatol 2011;55:1377-82.

49. Jitraruch S, Fitzpatrick E, Deheragoda M, et al. Autoimmune Liver Disease in Children with Sickle Cell Disease. J Pediatr 2017;189:79-85.e2.

50. Feld JJ, Kato GJ, Koh C, et al. Liver injury is associated with mortality in sickle cell disease. Aliment Pharmacol Ther 2015;42:912-21.

51. Azzu V, Fonseca M, Duckworth A, et al. Liver disease is common in patients with common variable immunodeficiency and predicts mortality in the presence of cirrhosis or portal hypertension. J Allergy Clin Immunol Pract 2019;7:2484-6.e3.

52. Resnick ES, Moshier EL, Godbold JH, et al. Morbidity and mortality in common variable immune deficiency over 4 decades. Blood 2012;119:1650-7.

53. Cunningham-Rundles C, Bodian C. Common variable immunodeficiency: clinical and immunological features of 248 patients. Clin Immunol 1999;92:34-48.

54. Fuss IJ, Friend J, Yang Z, et al. Nodular regenerative hyperplasia in common variable immunodeficiency. J Clin Immunol 2013;33:748-58.

55. Malamut G, Ziol M, Suarez F, et al. Nodular regenerative hyperplasia: the main liver disease in patients with primary hypogammaglobulinemia and hepatic abnormalities. J Hepatol 2008;48:74-82.

56. Azzu V, Elias JE, Duckworth A, et al. Liver transplantation in adults with liver disease due to common variable immunodeficiency leads to early recurrent disease and poor outcome. Liver Transpl 2018;24:171-81.

57. Ben-Yakov G, Kapuria D, Marko J, et al. Liver disturbances in activated phosphoinositide 3-kinase delta syndrome. J Allergy Clin Immunol Pract 2018;6:1763-5.

58. Grunebaum E, Avitzur Y. Liver-associated immune abnormalities. Autoimmun Rev 2019;18:15-20.

59. Hussain N, Feld JJ, Kleiner DE, et al. Hepatic abnormalities in patients with chronic granulomatous disease. Hepatology 2007;45:675-83.

60. Feld JJ, Hussain N, Wright EC, et al. Hepatic Involvement and Portal Hypertension Predict Mortality in Chronic Granulomatous Disease. Gastroenterology 2008;134:1917-26.

61. Zhou Q, Yang D, Ombrello AK, et al. Early-onset stroke and vasculopathy associated with mutations in ADA2. N Engl J Med 2014;370:911-20.

62. Barron K, Ombrello A, Stone D, et al. The clinical spectrum of the deficiency of adenosine deaminase 2 (DADA2) continues to expand. 10th Congress of International Society of Systemic Auto-Inflammatory Diseases (ISSAID). Pediatr Rheumatol 2019. doi: 10.1186/ s12969-019-0313-x.

63. Kapuria D, Ben-Yakov G, Ortolano R, et al. The Spectrum of Hepatic Involvement in Patients With Telomere Disease. Hepatology 2019;69:2579-85.

64. Calado RT, Regal JA, Kleiner DE, et al. A spectrum of severe familial liver disorders associate with telomerase mutations. PLoS One 2009;4:e7926.

65. Álamo JM, Bernal C, Barrera L, et al. Liver Transplantation in Patients With Cryptogenic Cirrhosis: Long-Term Follow-up. Transplant Proc 2011;43:2230-2.

66. Schouten JN, Garcia-Pagan JC, Valla DC, et al. Idiopathic noncirrhotic portal hypertension. Hepatology 2011;54:1071-81.

67. Schouten JN, Verheij J, Seijo S. Idiopathic non-cirrhotic portal hypertension: a review. Orphanet J Rare Dis 2015;10:67.

68. Riggio O, Gioia S, Pentassuglio I, et al. Idiopathic noncirrhotic portal hypertension: current perspectives. Hepat Med 2016;8:81-8.

69. Wanless IR, Godwin TA, Allen F, et al. Nodular regenerative hyperplasia of the liver in hematologic disorders: a possible response to obliterative portal venopathy. A morphometric study of nine cases with an hypothesis on the pathogenesis. Medicine (Baltimore) 1980;59:367-79.

70. Nakanuma Y, Hoso M, Sasaki M, et al. Histopathology of the liver in non-cirrhotic portal hypertension of unknown aetiology. Histopathology 1996;28:195-204.

71. Zuo C, Chumbalkar V, Ells PF, et al. Prevalence of histological features of idiopathic noncirrhotic portal hypertension in general population: a retrospective study of incidental liver biopsies. Hepatol Int 2017;11:452-60.

72. Verheij J, Schouten JN, Komuta M, et al. Histological features in western patients with idiopathic non-cirrhotic portal hypertension. Histopathology 2013;62:1083-91. 
73. Kage M. Pathology of idiopathic non-cirrhotic portal hypertension. Hepatol Int 2017;11:409-11.

74. De Boer NK, Tuynman H, Bloemena E, et al. Histopathology of liver biopsies from a thiopurinenaive inflammatory bowel disease cohort: prevalence of nodular regenerative hyperplasia. Scand J Gastroenterol 2008;43:604-8.

75. Maida I, Garcia-Gasco P, Sotgiu G, et al. Antiretroviralassociated portal hypertension: a new clinical condition? Prevalence, predictors and outcome. Antivir Ther 2008;13:103-7.

76. Vilarinho S, Sari S, Yilmaz G, et al. Recurrent recessive mutation in deoxyguanosine kinase causes idiopathic noncirrhotic portal hypertension. Hepatology 2016;63:1977-86.

77. Koot BG, Alders M, Verheij J, et al. A de novo mutation in $\mathrm{KCNN} 3$ associated with autosomal dominant idiopathic non-cirrhotic portal hypertension. J Hepatol 2016;64:974-7.

78. Chitturi S, Abeygunasekera S, Farrell GC, et al. NASH and insulin resistance: Insulin hypersecretion and specific association with the insulin resistance syndrome. Hepatology 2002;35:373-9.

79. Marchesini G, Forlani G. NASH: From liver diseases to metabolic disorders and back to clinical hepatology. Hepatology 2002;35:497-9.

80. Hakim A, Zhang X, DeLisle A, et al. Clinical utility of genomic analysis in adults with idiopathic liver disease. J Hepatol 2019;70:1214-21.

81. Buzzetti E, Pinzani M, Tsochatzis EA. The multiple-hit pathogenesis of non-alcoholic fatty liver disease (NAFLD). Metabolism 2016;65:1038-48.

82. Lomonaco R, Ortiz-Lopez C, Orsak B, et al. Role of ethnicity in overweight and obese patients with nonalcoholic steatohepatitis. Hepatology 2011;54:837-45.

83. Younes R, Bugianesi E. NASH in Lean Individuals. Semin Liver Dis 2019;39:86-95.

84. Feng RN, Du SS, Wang C, et al. Lean-non-alcoholic fatty liver disease increases risk for metabolic disorders in a normal weight Chinese population. World J Gastroenterol 2014;20:17932-40.

85. WHO Expert Consultation. Appropriate body-mass index for Asian populations and its implications for policy and intervention strategies. Lancet 2004;363:157-63.

86. Younossi ZM, Stepanova M, Negro F, et al. Nonalcoholic fatty liver disease in lean individuals in the United States. Medicine (Baltimore) 2012;91:319-27.
87. Fan JG, Kim SU, Wong VWS. New trends on obesity and NAFLD in Asia. J Hepatol 2017;67:862-73.

88. Sinn DH, Gwak GY, Park HN, et al. Ultrasonographically detected non-alcoholic fatty liver disease is an independent predictor for identifying patients with insulin resistance in non-obese, non-diabetic middle-aged Asian adults. Am J Gastroenterol 2012;107:561-7.

89. Seto WK, Yuen MF. Nonalcoholic fatty liver disease in Asia: emerging perspectives. J Gastroenterol 2017;52:164-74.

90. Chen CH, Huang MH, Yang JC, et al. Prevalence and risk factors of nonalcoholic fatty liver disease in an adult population of taiwan: metabolic significance of nonalcoholic fatty liver disease in nonobese adults. J Clin Gastroenterol 2006;40:745-52.

91. Das K, Das K, Mukherjee PS, et al. Nonobese population in a developing country has a high prevalence of nonalcoholic fatty liver and significant liver disease. Hepatology 2010;51:1593-602.

92. Kim NH, Kim JH, Kim YJ, et al. Clinical and metabolic factors associated with development and regression of nonalcoholic fatty liver disease in nonobese subjects. Liver Int 2014;34:604-11.

93. Nishioji K, Sumida Y, Kamaguchi M, et al. Prevalence of and risk factors for non-alcoholic fatty liver disease in a non-obese Japanese population, 2011-2012. J Gastroenterol 2015;50:95-108.

94. Argo CK, Caldwell SH. Epidemiology and natural history of non-alcoholic steatohepatitis. Clin Liver Dis 2009; 13:511-31.

95. McPherson S, Hardy T, Henderson E, et al. Evidence of NAFLD progression from steatosis to fibrosing-steatohepatitis using paired biopsies: Implications for prognosis and clinical management. J Hepatol 2015;62:1148-55.

96. Fazel Y, Koenig AB, Sayiner M, et al. Epidemiology and natural history of non-alcoholic fatty liver disease. Metabolism 2016;65:1017-25.

97. Fracanzani AL, Valenti L, Bugianesi E, et al. Risk of nonalcoholic steatohepatitis and fibrosis in patients with nonalcoholic fatty liver disease and low visceral adiposity. J Hepatol 2011;54:1244-9.

98. Leung JC-F, Loong TC-W, Wei JL, et al. Histological severity and clinical outcomes of nonalcoholic fatty liver disease in nonobese patients. Hepatology 2017;65:54-64.

99. Kumar R, Rastogi A, Sharma MK, et al. Clinicopathological characteristics and metabolic profiles of non-alcoholic fatty liver disease in Indian patients 
with normal body mass index: Do they differ from obese or overweight non-alcoholic fatty liver disease? Indian J Endocrinol Metab 2013;17:665-71.

100.Cruz ACD, Bugianesi E, George J, et al. 379 Characteristics and Long-Term Prognosis of Lean Patients With Nonalcoholic Fatty Liver Disease. Gastroenterology 2014;146:S909.

101. Valenti L, Pelusi S, Baselli G. Whole exome sequencing for personalized hepatology: Expanding applications in adults and challenges. J Hepatol 2019;71:849-50.

102.Hakim A, Mistry PK, Vilarinho S. Reply to: "Whole exome sequencing for personalized hepatology: Expanding applications in adults and challenges". J Hepatol 2019;71:850-1.

103. Dyson JK, Beuers U, Jones DEJ, et al. Primary sclerosing cholangitis. Lancet 2018;391:2547-59.

104. Karlsen TH, Folseraas T, Thorburn D, et al. Primary sclerosing cholangitis - a comprehensive review. J Hepatol 2017;67:1298-323.

105.Lazaridis KN, LaRusso NF. Primary Sclerosing Cholangitis. N Engl J Med 2016;375:2501-2.

106. Sanchez-Valle A, Kassira N, Varela VC, et al. Biliary Atresia: Epidemiology, Genetics, Clinical Update, and Public Health Perspective. Adv Pediatr 2017;64:285-305.

107.Spada M, Riva S, Maggiore G, et al. Pediatric liver transplantation. World J Gastroenterol 2009;15:648-74.

108. Nault JC, Datta S, Imbeaud S, et al. Recurrent AAV2related insertional mutagenesis in human hepatocellular carcinomas. Nat Genet 2015;47:1187-93.

109. Romeo S, Kozlitina J, Xing C, et al. Genetic variation in PNPLA3 confers susceptibility to nonalcoholic fatty liver disease. Nat Genet 2008;40:1461-5.

110. Tian C, Stokowski RP, Kershenobich D, et al. Variant in PNPLA3 is associated with alcoholic liver disease. Nat Genet 2010;42:21-3.

111. Stender S, Kozlitina J, Nordestgaard BG, et al. Adiposity amplifies the genetic risk of fatty liver disease conferred by multiple loci. Nat Genet 2017;49:842-7.

112.Abul-Husn NS, Cheng X, Li AH, et al. A ProteinTruncating HSD17B13 Variant and Protection from Chronic Liver Disease. N Engl J Med 2018;378:1096-106.

113. Vilarinho S, Mistry PK. Exome Sequencing in Clinical Hepatology. Hepatology 2019;70:2185-92.

114. Goel A, Elias JE, Eapen CE, et al. Idiopathic NonCirrhotic Intrahepatic Portal Hypertension (NCIPH)Newer Insights into Pathogenesis and Emerging Newer Treatment Options. J Clin Exp Hepatol 2014;4:247-56.
115. Bloom S, Kemp W, Nicoll A, et al. Liver stiffness measurement in the primary care setting detects high rates of advanced fibrosis and predicts liver-related events in hepatitis C. J Hepatol 2018;69:575-83.

116. Wehr C, Kivioja T, Schmitt C, et al. The EUROclass trial: defining subgroups in common variable immunodeficiency. Blood 2008;111:77-85.

117.Laharie D, Vergniol J, Bioulac-Sage P, et al. Usefulness of noninvasive tests in nodular regenerative hyperplasia of the liver. Eur J Gastroenterol Hepatol 2010;22:487-93.

118. Vuppalanchi R, Mathur K, Pyko M, et al. Liver Stiffness Measurements in Patients with Noncirrhotic Portal Hypertension-The Devil Is in the Details. Hepatology 2018;68:2438-40.

119. Gioia S, Nardelli S, Pasquale C, et al. Natural history of patients with non cirrhotic portal hypertension: Comparison with patients with compensated cirrhosis. Dig Liver Dis 2018;50:839-44.

120.Da BL, Surana P, Kapuria D, et al. Portal Pressure in Non-Cirrhotic Portal Hypertension: To Measure or Not to Measure. Hepatology 2019;70:2228-30.

121. Bissonnette J, Genereux A, Cote J, et al. Hepatic hemodynamics in 24 patients with nodular regenerative hyperplasia and symptomatic portal hypertension. J Gastroenterol Hepatol 2012;27:1336-40.

122.Seijo S, Reverter E, Miquel R, et al. Role of hepatic vein catheterisation and transient elastography in the diagnosis of idiopathic portal hypertension. Dig Liver Dis 2012;44:855-60.

123. Rodrigues SG, Montani M, Guixe-Muntet S, et al. Patients With Signs of Advanced Liver Disease and Clinically Significant Portal Hypertension Do Not Necessarily Have Cirrhosis. Clin Gastroenterol Hepatol 2019;17:2101-9.e1.

124. Sharma P, Agarwal R, Dhawan S, et al. Transient Elastography (Fibroscan) in Patients with Non-cirrhotic Portal Fibrosis. J Clin Exp Hepatol 2017;7:230-4.

125.Etzion O, Takyar V, Novack V, et al. Spleen and Liver Volumetrics as Surrogate Markers of Hepatic Venous Pressure Gradient in Patients With Noncirrhotic Portal Hypertension. Hepatol Commun 2018;2:919-28.

126.Armstrong K, Ranganathan R, Fishman M. Toward a Culture of Scientific Inquiry — The Role of Medical Teaching Services. N Engl J Med 2018;378:1-3.

127. Splinter K, Adams DR, Bacino CA, et al. Effect of Genetic Diagnosis on Patients with Previously Undiagnosed Disease. N Engl J Med 2018;379:2131-9.

128. Vilarinho S, Choi M, Jain D, et al. Individual exome 


\section{Page 14 of 14}

analysis in diagnosis and management of paediatric liver failure of indeterminate aetiology. J Hepatol 2014;61:1056-63.

129. Wenger AM, Guturu H, Bernstein JA, et al. Systematic

doi: $10.21037 /$ tgh.2020.04.04

Cite this article as: Gao E, Hercun J, Heller T, Vilarinho S. Undiagnosed liver diseases. Transl Gastroenterol Hepatol 2021;6:28.
Translational Gastroenterology and Hepatology, 2021

reanalysis of clinical exome data yields additional diagnoses: implications for providers. Genet Med 2017;19:209-14. 